\title{
CORRIGENDUM
}

\section{A protein transistor made of an antibody molecule and two gold nanoparticles}

Yu-Shiun Chen, Meng-Yen Hong and G. Steven Huang

Nature Nanotechnology 7, 197-203 (2012); published online 26 February 2012; corrected after print 2 March 2012

In the version of this Article originally published, the address of the first affiliation was incorrect; the correct address should have read 'Biomedical Electronics Translational Research Center, National Chiao Tung University, 1001 University Road, Hsinchu, Taiwan, ROC'. This has now been corrected in the HTML and PDF versions. 